\section{Solar wind implantation supplied light volatiles during the first stage of Earth accretion}

\section{S. Péron ${ }^{1 *}$, M. Moreira ${ }^{1}$, B. Putlitz² ${ }^{2}$ M.D. Kurz ${ }^{3}$}

open 2 AcCESS

\section{Abstract}

The isotopic and elemental compositions of noble gases constitute a powerful tool to study volatile origin and evolution, due to their inertness, and can thus provide crucial information about the early stage of planetary formation. Two models are proposed to explain the light noble gas origin on Earth: the solar wind implantation model and the solar nebula gas dissolution model. However, noble gas measurements often show addition of air to the mantle-derived gas, which complicates the determination of mantle isotopic ratios. We analysed the noble gas isotopic compositions of single vesicles in samples from the Galápagos hotspot with laser ablation, in order to understand and remove this atmospheric component, as well as discriminate between the two scenarios. Based on the new high precision results and a new statistical approach, we show that the solar wind implantation model is more likely to explain the terrestrial He, Ne and Ar the som

constraints on the solar system environment during the early stage of planetary formation.

Received 12 October 2016 | Accepted 8 February 2017 | Published 13 March 2017

\section{Introduction}

The isotopes ${ }^{20} \mathrm{Ne},{ }^{22} \mathrm{Ne},{ }^{36} \mathrm{Ar}$ and ${ }^{38} \mathrm{Ar}$ are primordial in the Earth's mantle, the ${ }^{20} \mathrm{Ne} /{ }^{22} \mathrm{Ne}$ and ${ }^{38} \mathrm{Ar} /{ }^{36} \mathrm{Ar}$ isotopic ratios can thus be used as noble gas source tracers. The major noble gas carriers in the solar system are, among others, the solar wind $\left({ }^{20} \mathrm{Ne} /{ }^{22} \mathrm{Ne}=13.8-14.0,{ }^{38} \mathrm{Ar} /{ }^{36} \mathrm{Ar}=0.1818-0.1828\right.$; Heber et al., 2009; Pepin et al., 2012), the component B $\left({ }^{20} \mathrm{Ne} /{ }^{22} \mathrm{Ne}=12.5-12.7,{ }^{38} \mathrm{Ar} /{ }^{36} \mathrm{Ar}=0.1862\right.$ or 0.1919; Black, 1972; Raquin and Moreira, 2009; Moreira, 2013; Moreira and

1. Institut de Physique du Globe de Paris - Sorbonne Paris Cité, UMR CNRS 7154, Université Paris Diderot, 1 Rue Jussieu, 75005 Paris, France

Corresponding author (email: peron@ipgp.fr)

2. Institute of Earth Sciences, University of Lausanne, Géopolis Building, CH-1015 Lausanne, Switzerland

3. Marine Chemistry and Geochemistry, MS \#25, Clark 421, Woods Hole Oceanographic Institution, Woods Hole, MA 02543, United State

Charnoz, 2016), which corresponds to solar wind implantation and is mostly important for light noble gases, and the phase $\mathrm{Q}$ for heavy noble gases found in chondrites $\left({ }^{20} \mathrm{Ne} /{ }^{22} \mathrm{Ne}=10.1-10.7,{ }^{38} \mathrm{Ar} /{ }^{36} \mathrm{Ar}=0.1873\right.$; Busemann et al., 2000). The lower mantle is one plausible reservoir of primitive noble gases (Allègre $e t$ al., 1983), so some Oceanic Island Basalts (OIBs), which are presumed to sample the lower mantle, are of particular interest to determine the Earth's primordial isotopic ratios. Only a limited suite of samples from a few hotspots (Hawaii, Galápagos and Iceland) are suitable because unradiogenic/primitive ( $\mathrm{He}, \mathrm{Ne})$ isotopic compositions are rare, and because submarine or subglacial glass samples are needed to analyse the primitive noble gas compositions. Such glass samples correspond to magma quenched at high pressures during eruptions and are among the best samples to record the mantle noble gas composition.

However, the ubiquity of an atmospheric component has complicated the determination of mantle source noble gas isotopic compositions. The air component may be derived from post-eruption contamination when samples are recovered from the seafloor (Ballentine and Barfod, 2000). It may also come from atmospheric recycling through subduction for heavy noble gases ( $\mathrm{Ar}, \mathrm{Kr}, \mathrm{Xe}$ ) (Holland and Ballentine, 2006; Kendrick et al., 2011; Parai and Mukhopadhyay, 2015). Noble gas studies often use step-crushing to analyse samples, and the results yield mixing trends between an atmospheric end-member and a mantle end-member. It is then assumed that the highest measured value is the least air contaminated and corresponds to a lower limit for the mantle. However, this approach does not necessarily completely eliminate atmospheric contamination. In order to avoid the atmospheric component, laser ablation analyses were introduced (Burnard et al., 1997; Burnard, 1999), which involve targeting individual bubbles with a laser. Up to now, no laser ablation study allows us to clearly discriminate between the two scenarios for noble gas origin on Earth because of the scarcity of data and the poor accuracy (Raquin et al., 2008; Colin et al., 2015; Péron et al., 2016).

We analysed by laser ablation the noble gas compositions ( $\mathrm{He}, \mathrm{Ne}, \mathrm{Ar}$ ) of twenty vesicles of two submarine glass samples from Fernandina volcano (Galápagos), previously imaged via X-ray microtomography to locate the vesicles (Supplementary Information and Table S-1), with the aim of understanding the air contamination mechanisms, and refining the Earth's primordial ${ }^{20} \mathrm{Ne} /{ }^{22} \mathrm{Ne}$ and ${ }^{38} \mathrm{Ar} /{ }^{36} \mathrm{Ar}$ ratios to evaluate the two models for noble gas origin on Earth; the solar wind implantation model (Trieloff et al. 2000: Raquin and Moreira, 2009) and the solar nebula gas dissolution model (Yokochi and Marty, 2004; Mukhopadhyay, 2012). These OIB samples, AHA-NEMO2-D22A and AHA-NEMO2D22B, were chosen due to their very primitive $\mathrm{He}$ and Ne compositions (Kurz et al., 2009), relatively high gas concentrations and thick glassy rinds (results are in Tables S-2 and S-3). 


\section{Evidence for a Ne-B Component in the Mantle}

All the new vesicles analysed show similar isotopic compositions, with only two exceptions (vesicles V4B and V16B; Figs. 1, 2 and Table S-3). Vesicles V4B and V16B may be contaminated by air since their ${ }^{20} \mathrm{Ne} /{ }^{22} \mathrm{Ne}$ and ${ }^{40} \mathrm{Ar} /{ }^{36} \mathrm{Ar}$ ratios are lower and tend to atmospheric ratios. Small but subtle cracks are observed just above these bubbles on the X-ray microtomography images (Figs. S-1, S-2, S-3 and $\mathrm{S}-4$ ) and must have been hit before piercing these vesicles, mixing air with vesicle gases. This is a new evidence of atmospheric contamination mechanisms. These cracks, typically less than $20 \mu \mathrm{m}$ wide, are difficult to notice. Except those two vesicles, and the vesicles for which the uncertainties are large (V2B, V5B, V6B $\mathrm{V} 11 \mathrm{~B}$ and $\mathrm{V} 2 \mathrm{~A})$, the ${ }^{20} \mathrm{Ne} /{ }^{22} \mathrm{Ne}$ ratio is between $12.17 \pm 0.13$ and $12.83 \pm 0.09(1 \sigma)$, the vesicles have a ${ }^{38} \mathrm{Ar} /{ }^{36} \mathrm{Ar}$ ratio similar to air (0.188) and the ${ }^{40} \mathrm{Ar} /{ }^{36} \mathrm{Ar}$ ratio is between $5809 \pm 425$ and $8206 \pm 695$ (Table S-3 and Fig. S-5).

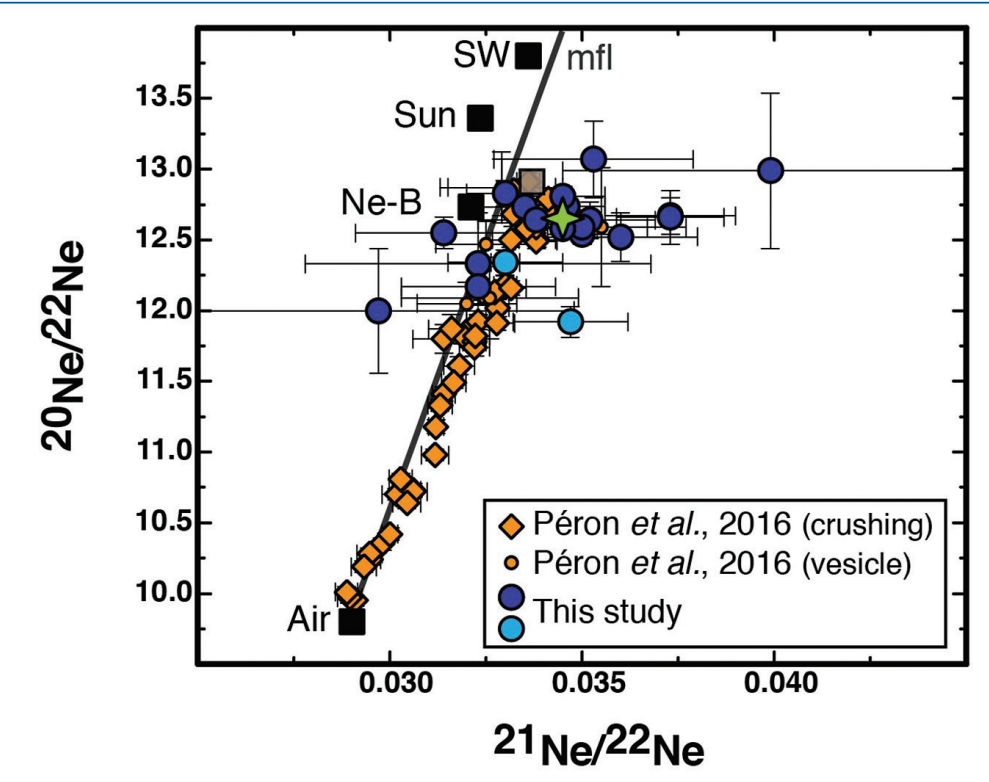

Figure 1 The three neon isotopes plot for the vesicles. Data from the literature for the same samples (Peron et al., 2016) are in orange diamonds and circles. The new data are in blue circles, the two vesicles in light blue (V4B and V16B) are assumed to be air contaminated (Figs. S-1, $\mathrm{S}-2)$. The Fernandina source isotopic composition estimated from the statistical analysis with the new data is indicated with the green star, in comparison with the previous one (Péron et al., 2016) indicated with the grey square. Ne-B (Moreira, 2013), Sun (Heber et al., 2012), solar wind SW (Heber et al., 2009), $\mathrm{mfl}$ mass fractionation line.

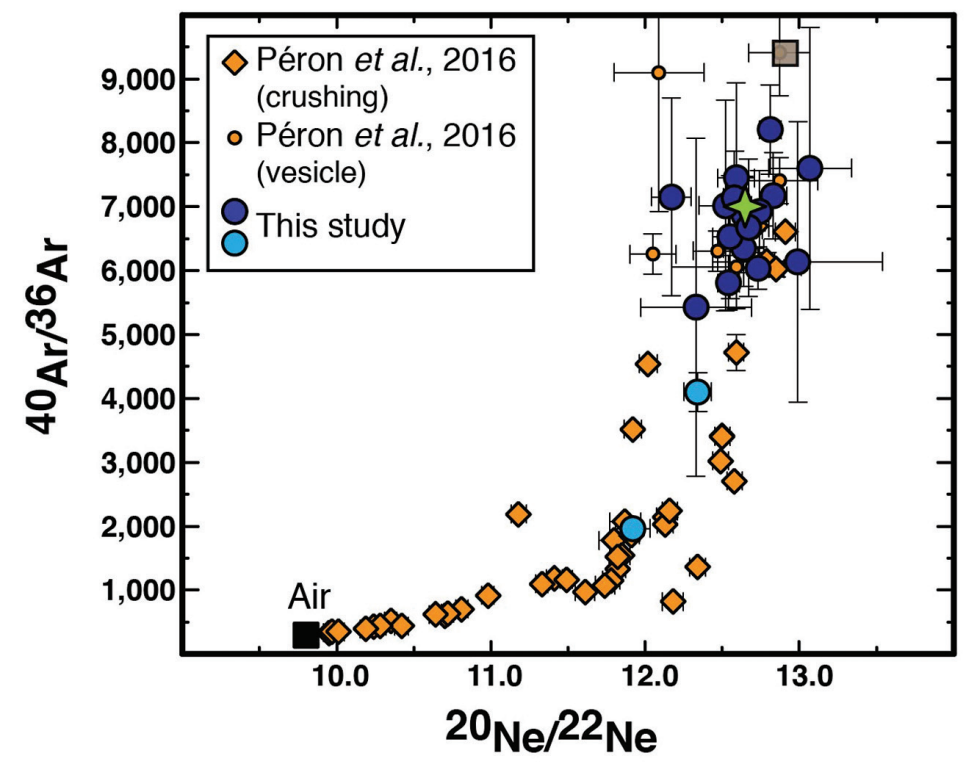

Figure $2 \quad{ }^{40} \mathrm{Ar} /{ }^{36} \mathrm{Ar}$ ratio $v \mathrm{vs}^{20} \mathrm{Ne} /{ }^{22} \mathrm{Ne}$ ratio for the vesicles. The latter are in blue circles (vesicles V2A and V11B have too large uncertainties and are not represented). Previous data are in orange diamonds and circles (Péron et al., 2016). The two vesicles in light blue (V4B and V16B) are assumed to be air contaminated, as indicated by the ${ }^{40} \mathrm{Ar} /{ }^{36} \mathrm{Ar}$ data, along with distribution of microcracks (Figs. S-1, S-2, S-3, S-4, S-8). The Fernandina source isotopic composition inferred from laser ablation data alone is indicated with the green star, in comparison with the previous one (Péron et al., 2016) indicated with the grey square.

The relatively large dataset (20 vesicles) combined with the fact that all the vesicles have similar isotopic compositions (Figs. 1 and 2), suggests that they al sample the same gas. This allows a statistical analysis (Fig. 3) in order to derive precisely the Fernandina source isotopic ratios. In this new approach, a Gaussian distribution is considered for each vesicle isotopic ratio, where the mean is the measured isotopic ratio and sigma is the associated measurement uncertainty, and then the sum of all these Gaussian distributions for a given isotopic ratio gives a cumulative curve. These cumulative curves are thus fitted to obtain overall means (Supplementary Information). This allows us to weight the mean according to measurement uncertainty. We use all the vesicles except the two contaminated ones (V4B and V16B). By fitting the curves with Gaussian distributions on Figure 3 (and Fig. S-6), we obtained a ${ }^{20} \mathrm{Ne} /{ }^{22} \mathrm{Ne}$ ratio of $12.65 \pm 0.04(\sigma / \sqrt{\mathrm{n}}), \mathrm{a}^{21} \mathrm{Ne} /{ }^{22} \mathrm{Ne}$ ratio of $0.0345 \pm 0.0004(\sigma / \sqrt{ } \mathrm{n})$ and ${ }^{38} \mathrm{Ar} /{ }^{36} \mathrm{Ar}$ ratio of $0.1887 \pm 0.0006(\sigma / \sqrt{ } \mathrm{n})$ The ${ }^{40} \mathrm{Ar} /{ }^{36} \mathrm{Ar}$ distribution could not be fitted with a Gaussian curve but the data show that this ratio is between 6000 and 7000 in the Fernandina source (Fig. S-7) 
a

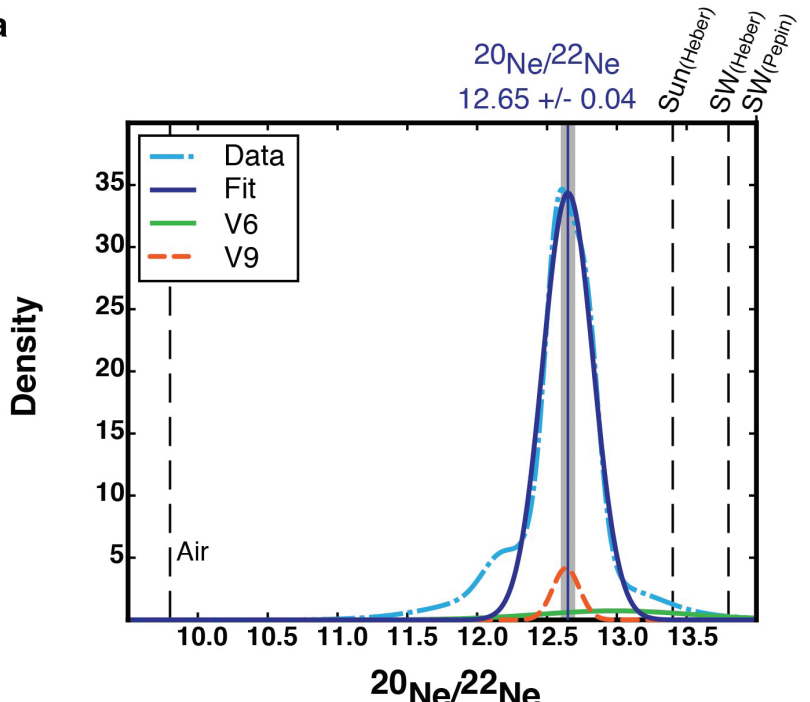

b

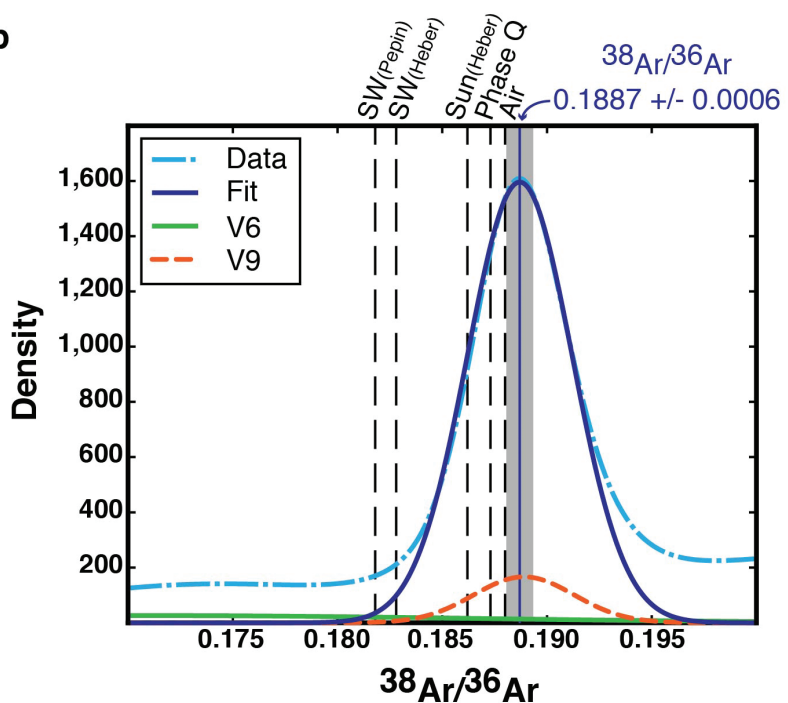

Figure 3 Gaussian curves obtained (a) for the ${ }^{20} \mathrm{Ne} / 22 \mathrm{Ne}$ ratio and (b) for the ${ }^{38} \mathrm{Ar} /{ }^{36} \mathrm{Ar}$ ratio The light blue dotted curve is the cumulative curve obtained with the data (except V4B and V16B), the dark blue curve is the Gaussian fit. As an example, the orange dotted curve for vesicle V9 contributes strongly to the cumulative curve, whereas V6 (green curve) is poorly constrained. The mean isotopic ratios, from the statistical analysis, are represented with the blue lines and the corresponding uncertainties $(\sigma / \sqrt{n})$ with the shaded areas. Solar wind (SW) (Heber et al., 2009; Pepin et al., 2012), Sun (Heber et al., 2012), Phase Q (Busemann et al., 2000).

\section{Discussion and Conclusions}

The new results are consistent with previous studies about the Hawaii and Iceland hotspots (Trieloff et al., 2000; Mukhopadhyay, 2012; Colin et al., 2015). The highest values of $12.91 \pm 0.07$ and $9407 \pm 672$ for the ${ }^{20} \mathrm{Ne} /{ }^{22} \mathrm{Ne}$ and ${ }^{40} \mathrm{Ar} /{ }^{36} \mathrm{Ar}$ ratios respectively obtained in a previous study of the same samples (Péron et al., 2016) seem to be slightly too high for the Fernandina mantle source within $2 \sigma$ uncertainties. Hence, it does not seem appropriate to take the highest values for determining mantle source isotopic ratios. We cannot exclude that isotopic fractionation occurs during bubble formation (Ruzié and Moreira, 2010), which could increase the ${ }^{20} \mathrm{Ne} / 22 \mathrm{Ne}$ ratio for a few bubbles, and also that slight sample heterogeneities exist, which could explain the small variability observed between the bubbles. It is thus more suitable to take a mean of the data, provided that the bubbles have similar compositions. The statistical analysis used here provides a new approach for precise determination of isotopic ratios.

As detailed in Supplementary Information, we do not expect contamination of the bubbles. Even if recycling of heavy noble gases ( $\mathrm{Ar}, \mathrm{Kr}, \mathrm{Xe})$ through subduction may occur (Holland and Ballentine, 2006; Kendrick et al., 2011; Parai and Mukhopadhyay, 2015), recycling of $\mathrm{He}$ and $\mathrm{Ne}$ into the OIB gas-rich source is not significant (Staudacher and Allègre, 1988; Holland and Ballentine, 2006). The ${ }^{20} \mathrm{Ne} /{ }^{22} \mathrm{Ne}$ ratio of $12.65 \pm 0.04$ is thus likely to represent the Fernandina source isotopic composition.

The ${ }^{20} \mathrm{Ne} /{ }^{22} \mathrm{Ne}$ ratio corresponds quite closely to the Ne-B composition. The Ne-B ${ }^{20} \mathrm{Ne} / 22 \mathrm{Ne}$ ratio was determined from analyses of gas-rich meteorites and lunar soils, which have been exposed to solar wind irradiation for millions of years (12.52; Black, 1972; 12.8 on average; Eberhardt et al., 1972), and also from numerical simulations (12.73; Raquin and Moreira, 2009; Moreira, 2013; Moreira and Charnoz, 2016). This is the first high precision laser ablation study for $\mathrm{Ne}$, involving enough vesicles for a statistical analysis. The fact that all the vesicles show similar isotopic compositions, in the Ne-B range of values, combined with the lunar soil results, is a strong argument in favour of the solar wind implantation model to explain light noble gas origin on Earth. The new results are consistent with this steady-state (Ne-B) composition and it may not be necessary to invoke non-steady state solar wind implantation onto grains to explain the lower mantle ${ }^{20} \mathrm{Ne} / 22 \mathrm{Ne}$ ratio (Péron et al, 2016). Other studies suggest that the ${ }^{38} \mathrm{Ar} /{ }^{36} \mathrm{Ar}$ ratio is atmospheric in the Earth's mantle (Trieloff et al., 2000; Raquin and Moreira, 2009; Mukhopadhyay, 2012), and lunar soils seem to have $\mathrm{a}^{38} \mathrm{Ar} /{ }^{36} \mathrm{Ar}$ ratio close to the atmospheric value even if the resolution is low (e.g., Eberhardt et al., 1972; Benkert et al., 1993). Hence, the solar wind implantation model could also account for the lower mantle ${ }^{38} \mathrm{Ar} /{ }^{36} \mathrm{Ar}$ ratio.

Previous step-crushing studies advocated that the early Earth would have captured a primordial atmosphere from solar nebula gas and that noble gases come from the dissolution of this early atmosphere into a magma ocean (Yokochi and Marty, 2004; Mukhopadhyay, 2012). This scenario is problematic for several 
reasons. First, the mantle noble gas isotopic composition is different from the solar or solar wind compositions (the Sun ${ }^{20} \mathrm{Ne} / 22 \mathrm{Ne}$ ratio would be 13.34; Heber et al., 2012). The solar nebula dissolution model would thus require subduction of atmospheric $\mathrm{Ne}$ to lower the ${ }^{20} \mathrm{Ne} /{ }^{22} \mathrm{Ne}$ ratio from solar to the actual mantle value. However, Ne recycling into the Ne-rich, lower mantle is not significant (Staudacher and Allègre, 1988; Holland and Ballentine, 2006). Finally, this model requires that enough Ne would have been dissolved into the Earth's precursors (Mars-sized objects) because the solar nebula is blown in a few Ma (Wyatt et al., 2003), well before Earth accretion has finished, which may be problematic because those objects cannot capture dense atmospheres.

Implantation of solar wind ions onto grains has also been suggested to explain the very low $\mathrm{D} / \mathrm{H}$ ratio of the lower mantle (highly negative $\delta \mathrm{D}$ of $-218 \%$ o) measured in olivine-hosted melt inclusions of a Baffin Island sample (Hallis et al., 2015). The solar nebula $\delta \mathrm{D}$ ratio is $-870 \%$ ond so solar wind irradiation, a mechanism able to enrich grains in heavy isotopes, could explain the lower mantle value and could then be one of the sources of water on Earth (Hallis et al., 2015). This scenario offers a simple alternative to explain light volatiles $(\mathrm{H}, \mathrm{He}, \mathrm{Ne})$ origin on Earth.

This study has put forward two major implications. First, it is more reliable to consider a mean of laser ablation data rather than taking the highest measured values to determine precisely mantle source compositions. Secondly, this new approach supports the model of solar wind irradiation associated with sputtering to explain He and Ne origin on Earth. Solar wind implantation is also likely a major source of $\mathrm{Ar}$ and $\mathrm{H}$ on Earth.

\section{Acknowledgements}

We thank Lukas Baumgartner for the generous access to the tomography facilities. We also thank Déborah Chavrit, Frédéric Moynier, Alberto E. Saal and John Creech for their constructive comments and discussion on the first versions of this manuscript. We acknowledge the financial support from the UnivEarthS Labex program of Sorbonne Paris Cité (ANR-10-LABX-0023 and ANR-11-IDEX-0005-02). The image for the graphical abstract was designed by Joël Dyon of IPGP.

Editor: Bruce Watson

\section{Additional Information}

Supplementary Information accompanies this letter at www.geochemical perspectivesletters.org/article1718

Reprints and permission information are available online at http://www. geochemicalperspectivesletters.org/copyright-and-permissions
Cite this letter as: Péron, S., Moreira, M., Putlitz, B., Kurz, M.D. (2017) Solar wind implantation supplied light volatiles during the first stage of Earth accretion. Geochem. Persp. Let. 3, 151-159.

\section{References}

Allègre, C.J., Staudacher, T., SARDA, P., KuRz, M.D. (1983) Constraints on evolution of Earth's mantle from rare gas systematics. Nature 303, 762-766.

BALLEnTINe, C., BARFOD, D. (2000) The origin of air-like noble gases in MORB and OIB. Earth and Planetary Science Letters 180, 39-48.

BenKERT, J.-P., BAUR, H., Signer, P., Wieler, R. (1993) He, Ne and Ar from the Solar Wind and Solar Energetic Particles in lunar ilmenites and pyroxenes. Journal of Geophysical Research 98, $13147-13162$.

BLACK, D.C. (1972) On the origins of trapped helium, neon and argon isotopic variations in meteorites-I. Gas-rich meteorites, lunar soil and breccia. Geochimica et Cosmochimica Acta 36, 347-375.

BURNARD, P. (1999) The bubble-by-bubble volatile evolution of two mid-ocean ridge basalts. Earth and Planetary Science Letters 174, 199-211.

Burnard, P., Graham, D., TuRner, G. (1997) Vesicle-specific noble gas analyses of "popping rock": implications for primordial noble gases in the Earth. Science 276, 568-571.

Busemann, H., Baur, H., Wieler, R. (2000) Primordial noble gases in "phase Q" in carbonaceous and ordinary chondrites studied by closed-system stepped etching. Meteoritics and Planetary Science 35, 949-973.

Colin, A., Moreira, M., Gautheron, C., Burnard, P. (2015) Constraints on the noble gas composition of the deep mantle by bubble-by-bubble analysis of a volcanic glass sample from Iceland. Chemical Geology 417, 173-183.

Eberhardt, P., Geiss, J., Graf, H., Grögler, N., Mendia, M.D., Mörgeli, M., Schwaller, H., StettLeR, A., KRÄHEnbÜHL, U., Von Gunten, H.R. (1972) Trapped solar wind noble gases in Apollo 12 lunar fines 12001 and Apollo 11 breccia 10046. Proceedings of the Third Lunar Science Conference, Supplement 3, Geochimica et Cosmochimica Acta, The MIT press 2, 1821-1856.

Hallis, L.J., Huss, G.R., Nagashima, K., TAYlor, G.J., Halldórsson, S.A., Hilton, D.R, MotTL, M.J., MeECH, K.J. (2015) Evidence for primordial water in Earth's deep mantle. Science 350, 795-797

Heber, V.S., Wieler, R., Baur, H., Olinger, C., Friedmann, A., Burnett, D.S. (2009) Noble gas composition of the solar wind as collected by the Genesis mission. Geochimica et Cosmochimica Acta 73, 7414-7432.

Heber, V.S., Baur, H., Bochsler, P., McKeegan, K.D., Neugebauer, M., Reisenfeld, D.B., WieLER, R., WIENS, R.C. (2012) Isotopic Mass Fractionation of Solar Wind: Evidence from Fast and Slow Solar Wind Collected by the Genesis mission. The Astrophysical Journal 759, 121.

Holland, G., BALLENTINE, C.J. (2006) Seawater subduction controls the heavy noble gas composition of the mantle. Nature 441, 186-191.

Kendrick, M.A., ScAmbelluri, M., HondA, M., PhildipS, D. (2011) High abundances of noble gas and chlorine delivered to the mantle by serpentine subduction. Nature Geoscience 4, 807-812.

Kurz, M.D., Curtice, J., Fornari, D., Geist, D., Moreira, M. (2009) Primitive neon from the center of the Galápagos hotspot. Earth and Planetary Science Letters 286, 23-34.

MoREIRA, M. (2013) Noble gas constraints on the origin and evolution of Earth's volatiles. Geochemical Perspectives 2, 229-403. 
Moreira, M., CharNOZ, S. (2016) The origin of the neon isotopes in chondrites and on Earth. Earth and Planetary Science Letters 433, 249-256.

MukHopadHYAY, S. (2012) Early differentiation and volatile accretion recorded in deep mantle Neon and Xenon. Nature 486, 101-104

Parai, R., MukHopadhyay, S. (2015) The evolution of MORB and plume mantle volatile budgets: Constraints from fission Xe isotopes in Southwest Indian Ridge basalts. Geochemistry, Geophysics, Geosystems 16, 719-735.

Pepin, R.O., Schlutter, D.J., BeCKeR, R.H., ReIsenfeld, D.B. (2012) Helium, neon, and argon composition of the solar wind as recorded in gold and other Genesis collector materials. Geochiica Acta 89, 62-80.

Péron, S., Moreira, M., Colin, A., Arbaret, L., Putlitz, B., KurZ, M.D. (2016) Neon isotopic composition of the mantle constrained by single vesicle analyses. Earth and Planetary Science Letters 449, 145-154.

RAQUin, A., MoReirA, M. (2009) Atmospheric ${ }^{38} \mathrm{Ar} /{ }^{36} \mathrm{Ar}$ in the mantle: Implications for the nature of the terrestrial parent bodies. Earth and Planetary Science Letters 287, 551-558.

Raquin, A., Moreira, M., Guillon, F. (2008) He, Ne and Ar systematics in single vesicles: Mantle isotopic ratios and origin of the air component in basaltic glasses. Earth and Planetary Science isotopic ratios and origin of the air component in basaltic glasses. Earth and Planetary Science
Letters 274, 142-150.

RUZIÉ, L., MoReIRA, M. (2010) Magma degassing process during Plinian eruptions. Journal of Volcanology and Geothermal Research 192, 142-150.

StAudAcher, T., AllèGre, C.J. (1988) Recycling of oceanic crust and sediments: the noble gas subduction barrier. Earth and Planetary Science Letters 89, 173-183.

Trieloff, M., KunZ, J., Clague, D.A., Harrison, D., AllËgre, C.J. (2000) The nature of pristine noble gases in mantle plumes. Science 288, 1036-1038.

WYatT, M.C., DenT, W.R.F., GREAVES, J.S. (2003) SCUBA observations of dust around Lindroos stars: evidence for a substantial submillimetre disc population. Monthly Notices of the Royal Astronomical Society 342, 876-888.

YOKOCHI, R., MARTY, B. (2004) A determination of the neon isotopic composition of the deep mantle. Earth and Planetary Science Letters 225, 77-88. 